\title{
Point-of-View Mining and Cognitive Presence in MOOCs: A (Computational) Linguistics Perspective
}

\author{
Noureddine Elouazizi \\ Science Center for Learning and Teaching, University of British Columbia \\ Center for Teaching and Learning Technologies, University of British Columbia \\ Department of Linguistics, Simon Fraser University \\ Vancouver, British Columbia \\ Email: noureddine.elouazizi@ubc.ca
}

\begin{abstract}
This paper explores the cognitive presence of the learners in MOOCs through using a (computational) linguistic analysis of the learners' Point-of-View as an indicator for cognitive presence. The linguistic analysis of the written language as a medium of interaction by the students in the context of MOOCs shows hallmarks of cognitive disengagement and low cognitive presence by the learners.
\end{abstract}

\section{Introduction}

The popularity of Massive Open Online Courses (henceforth MOOCs) is expanding, and the perceived educational add-value of their scalability to the masses is growing. However, research shows that MOOCs do not generate enough opportunities for students' interaction and retention (see: Kizilcec et al (2013), Yang et al (2013), Rosé et al (2014), Wen et al (2014b). The large scale linguistic data that is generated by discussion boards, blogs, and other written language-based interaction tools that are/can be part of the MOOC technology infrastructure provides an unprecedented opportunity to study the dynamics of students' interaction, learning engagement, and ways in which critical valuable learning/teaching discourse is constructed around different knowledge topics.

This paper presents an exploratory approach to examine students' learning-based inquiry within the context of MOOCs through analyzing the linguistic aspects of Point-ofView as an indicator of cognitive presence. This approach is theoretically grounded in linguistics (natural syntax) and educationally understood along the lines of the Community of Inquiry Framework (Garrison et al (2000), Garrison et al (2001), Swan et al (2009)).

\section{Background}

2.1.Point-of-View: a linguistics perspective I assume that the structure and the interpretation of Point-of-View as a linguistic construct is detected and interpreted through its compositional parts. The compositional parts of Point-of-View include notions such as: subjectivity, belief mitigation, evidentiality and epistemic mood (Speas and Tenny 2003, Elouazizi 2014). The notion of subjectivity refers to the way a speaker expresses knowledge gained through personal/internal experience-"ontological subjectivity" (Searle 2004). The notion of (belief) mitigation is linguistically conveyed through the use of a specific set of epistemic parenthetical verbs such as: think, believe (Urmson (1952)).

In addition to the use of belief mitigation verbs, and subjectivity linguistic devices, a speaker's point of view is also indicated by the degree of their use of evidential verbs that carry epistemic propositional attitudes, such as: I feel, I sense, I see. A subject/speaker uses evidentiality linguistic devices (verbs, adverbs) to evaluate the degree of certainty in a proposition by matching the source of the information and the target of the information (Speas 2008).

In addition to these linguistic devices, Point-of-View can also be conveyed through the use of a set of epistemic mood and discourse adverbs. This includes adverbs such as: frankly, presumably, supposedly, probably, luckily, realistically. These adverbs provide additional information about the propositional attitude of the speaker.

Taken jointly, the combination of these linguistic constructs constitutes a lexical structure (lexicon), with a latent syntax of Point-of-View, and which can lend itself to the techniques of text mining and computational linguistic analysis. 


\subsection{Point-of-View: a learning perspective}

Defined in broad terms, learning events are the set of "activities" and "acts" that the learner engages in so as to ensure the acquisition, transfer and modification of knowledge, skills and beliefs (Skinner (1968), Piaget (1952), Gagné (1985), Mayer (1996)). These (learning) activities and acts could be internal (mental) or/and external (behaviours) and could include more than one cognitive modality for processing information. These modalities include: auditory modality, visual modality, haptic modality, and linguistic modality. Each of these cognitive modalities produces data (information) that can be studied to infer whether and how learning occurs.

My focus here is on the linguistic modality, and how it is used to interface the components of an educational/learning experience, as understood within the context of the Community of Inquiry model for learning (see: Garrison et al (2000) and Garrison et al (2001)). Perceived from the perspective of the Community of Inquiry (CoI) model, I propose that the analysis of Point-of-View is a way to examine the nature of the supporting discourse that is crucial in interfacing the social presence, the cognitive presence, and the teaching presence (as illustrated in the adapted Figure in 1).

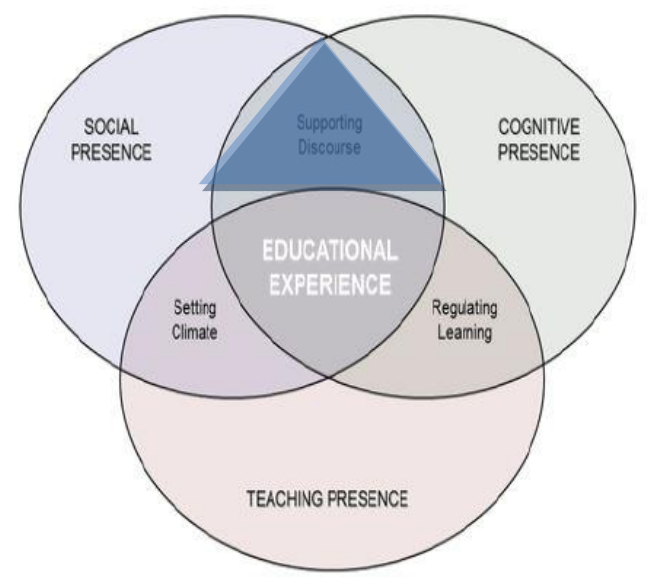

FIGURE 1: Community of Inquiry (CoI) model (Adapted from: Garrison et al 2000)

One of the central aspects of $\mathrm{CoI}$ is "cognitive presence". This refers to the "extent to which the participating learners in any particular configuration of a community of inquiry are able to construct meaning through sustained communication." (Garrison et al, Swan et al (2009)). I explore the cognitive presence of the MOOC's learners through the analysis of Point-of-View's usage, clustering and dispersions in the written language-based interactions, generated by the learners in the context of MOOCs.

\section{Method}

\subsection{Data sets: the corpus}

This paper uses two data sets of written language corpus. The first data set is from the blogs discussions of three MOOC courses, attended by 3000 learners, and English language was the language of interaction in these MOOCs. The second set of data comes from the discussion board of a large online university course, delivered to a large class of 300 students, using English language. The written corpus data from the three MOOCs contains 724955 words, and the corpus data from the non-MOOC online course contains 727205 words.

\begin{tabular}{l|c|c}
\hline $\begin{array}{c}\text { Lexical \& } \\
\text { Referential density }\end{array}$ & Three MOOCs & $\begin{array}{c}\text { One online } \\
\text { course }\end{array}$ \\
\hline Words in data set & 724955 & 727205 \\
\hline $\begin{array}{l}\text { Lexical density: } \\
\text { Lexemes per data set }\end{array}$ & 475064 & 381392 \\
\hline $\begin{array}{l}\text { Lexical density: } \\
\text { Lexemes \% }\end{array}$ & $61.17 \%$ & $52.91 \%$ \\
\hline $\begin{array}{l}\text { Reference density: } \\
1^{\text {st }} \text { Person (speaker) }\end{array}$ & $2.32 \%$ & $2.98 \%$ \\
\hline $\begin{array}{l}\text { Reference density: } \\
2^{\text {nd }} \text { Person (hearer) }\end{array}$ & $0.95 \%$ & 0.16 \\
\hline \# of learners & 3000 & 300
\end{tabular}

TABLE 1: lexical and referential density in the data sets

The data in table 1 was generated from the MOOC and the non-MOOC corpus data, using the corpus tool Systemic coder (available at: http://www.wagsoft.com/CorpusTool).

\subsection{Point-of-View extraction: the approach} One central non-trivial challenge with the computational extraction of the linguistic patterns from written text is the issue of classification and feature structures. The term classification is generally construed broadly to encompass the architecture and the structure of the systems and features used in extracting interpretative patterns such as opinions and sentiments from written text (Riloff and Wiebe (2003), Pang and Lee (2004)). There are different approaches to extraction to guide the computational process of automatically extracting patterns from text.

For example, the approach of polaritybased classification encompasses regression and ranking of the lexical units. This approach is exploited in sentiment analysis and it 
assumes that the text is underlined by an opinion towards which an agent expresses a positive or negative feeling (see: Pang et al. (2002), Eguchi and Lavrenko (2006)).

Another approach is the gradability-based classification. In this approach the lexical units are not attributed a polar classification. Rather, the text is classified in terms of gradable terms. This approach is used in automatic extraction of subjectivity from the text (Wiebe et al. (2001), Wilson et al. (2005), Yu and Hatzivassiloglou (2003)).

I build on the insights of polarity-based and gradability-based approaches to automatic extraction and processing of text and propose the classification in Figure 2.

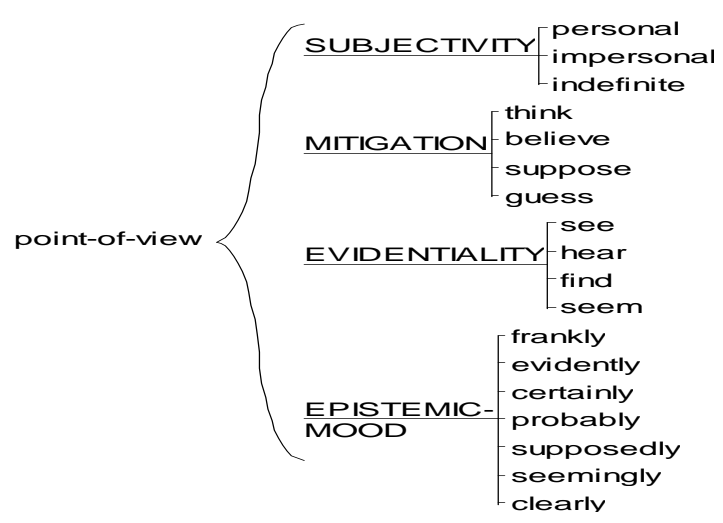

FIGURE 2: The classification features structure of Point-of-View

The classification and features structure in Figure 2 enables the extraction of a bundle of attitudes and belief as it uses a combination of parts of speech that encompass latent knowledge and interpretations, as exhibited in the syntax and the semantics of the constructs that compose Point-of-View. As such, Pointof-View mining and automatic analysis is at the intersection of opinion mining and subjectivity mining. This approach (Point-ofView mining) seeks to extract perspectiverelated information such as opinion holders, belief mitigation, and propositional attitudes (for similar but not identical approaches, see: Kudo and Matsumoto (2004), Dave et al (2003), Riloff and Wiebe (2003), Song et al 2007, El-Halees (2011)).

\section{Results and discussion}

Recall that the main goal of this paper is to explore the cognitive presence of the learners in MOOCs through their written language input, and using a linguistics analysis of the learners' Point-of-View as an indicator for cognitive presence. I hypothesize that if the learners in MOOCs are cognitively disengaged, the frequency and usage of Pointof-View components (as identified in Figure 2) would be low. Conversely, if the frequency and usage of Point-of-View components is higher, that would imply that the students in MOOCs are cognitively engaged and their cognitive presence is more asserted.

The Point-of-View usage data, as illustrated in FIGURE 3, shows that the use of belief mitigation devices (verbs such as: think, believe, guess and suppose) is equally low in all the three MOOCs.

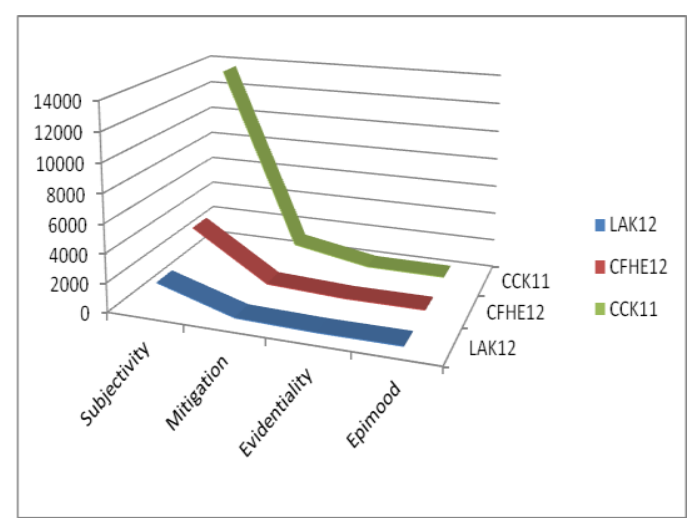

FIGURE 3: The usage of POV/cognitive presence in MOOCs

However, in all the three MOOCs, subjectivity is attested with varying degrees. This indicates that the learners do express their stance in their blog discussions but without engaging in any epistemic interactions with other learners. This is the case because the uses of the mitigation construct, of the evidentiality construct, and of the epistemic mood construct by a speaker (learner) always requires predicating on, hence interacting with previously mentioned/stated proposition. The data in Figure 3 indicates that the learners did not modify the propositions that were put forward by other learners or convey changes in propositional attitudes, or mitigate beliefs, expressed in the text of the MOOCs blogs.

These observations are further supported by the dispersion and clustering analysis of the data, as illustrated in Figure 4. 


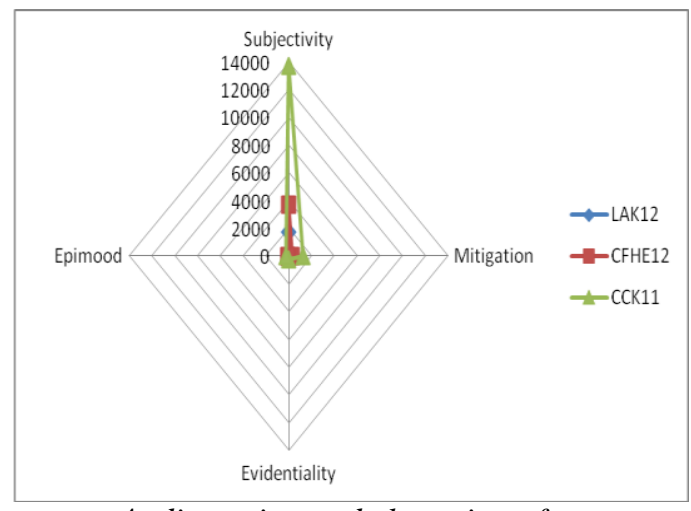

FIGURE 4: dispersion and clustering of POV/cognitive presence in MOOCs

Assuming that "support discourse" within the context of the CoI framework is crucial in interfacing the cognitive presence, the social presence and the teaching presence of the learner within an educational experience (see FIGURE 1 above), the use of written language as a medium of interaction by the students in the context of MOOCs shows hallmarks of cognitive disengagement and low cognitive presence. That this is indeed the case is further indicated by the Point-of-View comparative data that illustrates the use of Point-of-View in the context of a MOOC versus its use in the context of a non-MOOC online course.

Consider the differences in the Point-ofView usage, dispersion and clustering between the data set of a single MOOC ccourse and that of a non-MOOC online course, as illustrated by Figure 5 and Figure 6 below. The Point-ofView usage comparative data illustrated in Figure 5 indicates that the cognitive presence and engagement of the learners in the nonMOOC online course is significantly higher than in the MOOC courses.

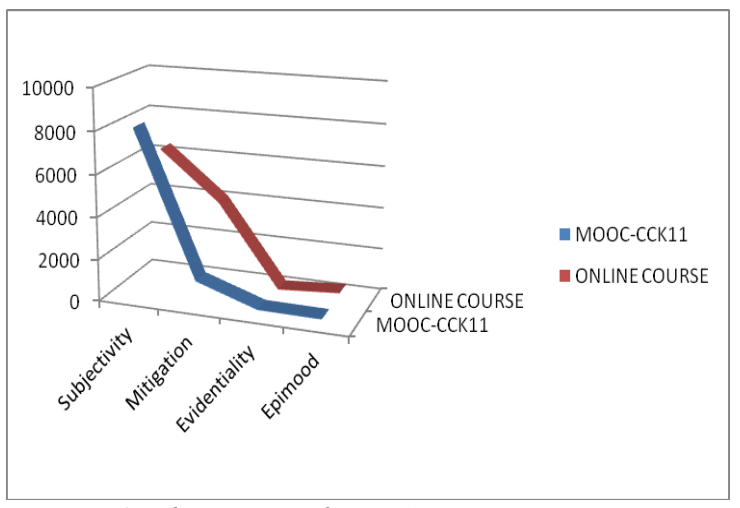

FIGURE 5: The usage of POV/cognitive presence in a MOOC vs. non-MOOC online course

The learners in the non-MOOC online course produced linguistic structures that contain speech acts of expressing subjective views, of mitigating aforementioned propositions (beliefs) in the discourse and of providing evidence of a statement as indicated through the use of evidential adverbs. However, the language (linguistic structures) used by the learners in a single MOOC course shows higher level of subjective use than the nonMOOC course but significantly lower usage of speech acts that express mitigation of beliefs or evidentiality.

Furthermore, the usage of the Point-ofView in the non-MOOC online course shows that the learners expressed higher subjectivity, accompanied with higher rates of belief mitigation. This suggests that the learners in the non-MOOC online course were more cognitively engaged and they actively engaged in invoking the discourse structures that support the interfacing of cognitive presence, teaching presence and social presence. These observations are further supported by the Point-of-View dispersion and clustering data represented in Figure 6.

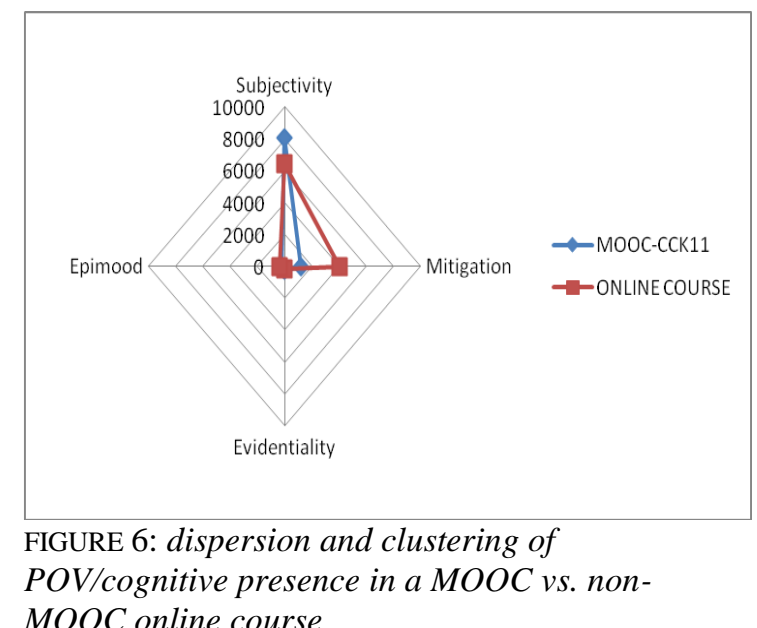

All in all, the data above indicates that learners within the context of MOOCs exhibit less cognitive presence than their counter part learners in a non-MOOC online course. The linguistic structures used by the learners in the context of a MOOC course, at least on the basis of the data examined in this paper, indicate that they did not mitigate and attach evidential statements to as many propositions as in a non-MOOC course. For a speaker to mitigate a proposition, the speaker first needs to be aware of the proposition, the agent who proposed or enacted such a proposition before mitigating it. Hence, the more a speaker mitigates hers or the propositions of others, the 
more the speaker is engaged with the discourse constructed around different knowledge topics.

These findings square with and confirm observations established in other studies which analyze the learners' engagement in MOOCs. For example, Wen et al. (2014a) and Wen et al (2014b), using a survival model, and drawing on linguistic data in discussion posts, show that learners' engagement in MOOCs reduces drastically after week three in a MOOC course.

\section{Conclusion and future work}

This paper explored the use of a computational linguistic perspective to mine and exploit the latent knowledge in the Point-of-View construct to examine the cognitive presence and engagement of the students in the context of MOOC and non-MOOC courses. As the results show, the linguistic analysis of the written language as a medium of interaction by the learners in the context of MOOCs shows hallmarks of cognitive disengagement and low cognitive presence by the learners.

However, what emerges in the context of this exploratory paper is a partial representation of the learning-based discourse structure within MOOCs and is by no means conclusive of the way discourse structures are constructed around different knowledge topics within the context of a MOOC vs. a nonMOOC online course. The empirical testing of the classification in Figure 2, from a text mining and automatic extraction perspective is yet to be validated on larger (fully) annotated MOOCs data sets, and using larger integrated lexicons that combine a Point-of-View latent knowledge lexicon and a MOOCs specific education experience lexicon.

\section{Acknowledgments}

I would like to thank Carolyn Penstein Rosé and George Siemens for sharing with me the MOOCs blog discussions data that I used as part of the data sets that this paper studies.

\section{References}

Dave, K., Lawrence, S., Pennock, D.M. 2003. Mining the peanut gallery: Opinion extraction and semantic classification of product reviews. In Proceedings of $W W W$, pages 519-528.
Elouazizi, Noureddine. 2014. The formal syntax of point-of-view and belief mitigation. $\mathrm{PhD}$ Dissertation, SFU.

El-Halees, A. 2011. Mining opinions in usergenerated contents to improve course evaluation. In Software Engineering and Computer Systems, pages 107-115. Springer.

Eguchi, K and Lavrenko, V.2006. Sentiment retrieval using generative models. In Proceedings of the Conference on Empirical Methods in Natural Language Processing (EMNLP), pages 345-354.

Gagné, R. M. 1985. The conditions of learning (4th ed.). New York: Holt, Rinehart \& Winston.

Garrison, D. R., Anderson, T, \& Archer, W. 2000. Critical inquiry in a text-based environment: Computer conferencing in higher education. The Internet and Higher Education 2: 87-105.

Garrison, D. R., Anderson, T., \& Archer, W. 2001. Critical thinking, cognitive presence and computer conferencing in distance education. American Journal of Distance Education, 15(1), 7-23.

Kizilcec, R. F., Piech, C. and Schneider, E. 2013. Deconstructing disengagement: analyzing learner subpopulations in massive open online courses. In Proceedings of the Third International Conference on Learning Analytics and Knowledge, pages 170-179.ACM.

Kudo, T. and Matsumoto, Y. 2004. A boosting algorithm for classification of semi-structured text. In Proceedings of the Conference on Empirical Methods in Natural Language Processing (EMNLP).

Mayer, R. E. 1996. Learners as information processors: Legacies and limitations of educational psychology's second metaphor. Educational Psychologist, 31, 151-161.

Pang, B., Lee, L. and Vaithyanathan, S. 2002. Thumbs up? Sentiment classification using machine learning techniques. In Proceedings of the Conference on Empirical Methods in Natural Language Processing (EMNLP), pages 79-86.

Pang, B. and Lee, L. 2004. A sentimental education: Sentiment analysis using subjectivity summarization based on minimum cuts. In Proceedings of the Association for Computational Linguistics (ACL), pages 271278. 
Piaget, J.-P. 1952. The origins of intelligence in children. International Universities Press, New York.

Rosé, C. P., Carlson, R., Yang, D., Wen, M., Resnick, L., Goldman, P. \& Sherer, J. 2014. Social Factors that Contribute to Attrition in MOOCs. In Proceedings of the First ACM Conference on Learning @ Scale(poster).

Swan, K., Garrison, D. R. \& Richardson, J. C. 2009. A constructivist approach to online learning: the Community of Inquiry framework. In Payne, C. R. (Ed.) Information Technology and Constructivism in Higher Education: Progressive Learning Frameworks. Hershey, PA: IGI Global, 43-57.

Song, D., Lin, H. and Yang. Z. 2007. Opinion mining in e-learning system. In Network and Parallel Computing Workshop. IFIP International Conference, P.788-792. IEEE.

Searle, J. 2003. Mind: a brief introduction. Oxford University Press.

Speas, Peggy. (2008). On the Syntax and Semantics of Evidentials. Language and Linguistics Compass. Volume 2 Issue 5, pp. 940 - 965.

Speas, Peggy, and Tenny, Carol. 2003. Configurational Properties of Point of View Roles. In DiSciullo, A. (ed.), Asymmetry in Grammar. Amsterdam: John Benjamins. 315344.

Urmson, J. O. 1952. Parenthetical verbs. Mind. 61. 480-496.

Wen, M., Yang, D., \& Rosé, C. P. 2014a. Sentiment Analysis in MOOC Discussion Forums: What does it tell us? In Proceedings of Educational Data Mining.

Wen, M., Yang, D., \& Rosé, C. P. 2014b. Linguistic Reflections of Student Engagement in Massive Open Online Courses. In Proceedings of the International Conference on Weblogs and Social Media.

Wiebe, J.M., Wilson, T and Bell, M. 2001. Identifying collocations for recognizing opinions. In Proceedings of the ACL/EACL Workshop on Collocation: Computational Extraction, Analysis, and Exploitation.

Wiebe, J. and Wilson, T. 2002. Learning to disambiguate potentially subjective expressions. In Proceedings of the Conference on Natural Language Learning (CoNLL), pages 112-118.
Wiebe., J., Theresa W. M., Bruce, R., Bell, M., and Martin, M. 2004. Learning subjective language. Computational Linguistics, 30(3):277-308.

Wilson, T., Wiebe, J. Hoffmann, P. 2005. Recognizing contextual polarity in phrase-level sentiment analysis. In Proceedings of the Human Language Technology Conference and the Conference on Empirical Methods in Natural Language Processing (HLT/EMNLP), pages 347-354.

Yang, D., Sinha, T., Adamson, D., \& Rosé, C. P. 2013. Turn on, Tune in, Drop out: Anticipating student dropouts in Massive Open Online Courses. In NIPS Data-Driven Education Workshop.

Yu, H., Hatzivassiloglou, V. 2003. Towards answering opinion questions: Separating facts from opinions and identifying the polarity of opinion sentences. In Proceedings of the Conference on Empirical Methods in Natural Language Processing (EMNLP). 